\title{
Epilepsy and suicide: pathogenesis, risk factors, and prevention
}

\author{
Alberto Verrotti' \\ Alessandra Cicconetti ${ }^{2}$ \\ Barbara Scorrano ${ }^{2}$ \\ Domenico De Berardis 2,3 \\ Carla Cotellessa ${ }^{2}$ \\ Francesco Chiarelli' \\ Filippo Maria Ferro ${ }^{2}$ \\ 'Department of Pediatrics; \\ ${ }^{2}$ Department of Oncology and \\ Neuroscience, Institute of Psychiatry, \\ University "G. d'Annunzio" of Chieti, \\ Italy; ${ }^{3}$ Department of Mental Health, \\ Teramo, Italy
}

\begin{abstract}
Depression and suicide tendencies are common in chronic diseases, especially in epilepsy and diabetes. Suicide is one of the most important causes of death, and is usually underestimated. We have analyzed several studies that compare mortality as a result of suicide in epileptic patients and in the general population. All the studies show that epileptic patients have a stronger tendency toward suicide than healthy controls. Moreover it seems that some kinds of epilepsy have a higher risk for suicide (temporal-lobe epilepsy). Among the risk factors are surgery therapy (suicide tendency five times higher than patients in pharmacological therapy), absence of seizures for a long time, especially after being very frequent, and psychiatric comorbidity (major depression, anxiety-depression disorders, personality disorders, substance abuse, psychoses). The aim of the review was to analyze the relationship between suicide and epilepsy, to identify the major risk factors, and to analyze effective treatment options.
\end{abstract}

Keywords: epilepsy, suicide, depression

\section{Introduction}

Although clinicians and researchers have documented an association between epilepsy and psychiatric disorders for more than 30 years (Ott et al 2003), depression and other disorders remain underdiagnosed and undertreated in young people with epilepsy (Suris et al 1996; Plioplys 2003). One recent study of children of 5 to 16 years found that although about $60 \%$ of them had a Diagnostic and Statistical Manual of Mental Disorders IV (DSM) psychiatric diagnosis, only about 33\% were receiving an appropriate psychiatric treatment (Ott et al 2003).

A paucity of published material has addressed the problem of suicide among epileptics. Studies on this topic have increased in recent years, but comparisons are difficult because of the variety of study designs, different criteria, and different populations studied.

This study's principal aims were to discuss the epidemiological data on suicide among epileptics, to analyze in depth the possible risk factors of suicide behavior in epileptic patients, and to try and develop prevention strategies for this important issue.

\section{Epidemiological data}

Suicide is a rare event, occurring only slightly more than once among 10,000 persons annually in the United States (Goodwin and Jamison 1990; Murphy 1994). A high suicide rate among patients with epilepsy has been documented. A Danish study (Henriksen et al 1970) showed that 164 of 2763 patients with epilepsy died (an excess mortality rate of $273 \%$ compared with the expected number of deaths in Denmark); the case material included all adult epileptics. Although suicide is probably not a major cause of death among epileptics (Hauser et al 1980; Cockell et al 1994), a number of investigations have reported rates of death due to suicide among individuals with epilepsy. The percentage of deaths by suicide ranges from $1 \%$ to $33 \%$ with an
Correspondence:Alberto Verrotti Department of Pediatry, University "G d'Annunzio" of Chieti, Italy Tel +3987 I3580I5

Fax +3987 I57 483।

Email averrott@unich.it 
overall average of about $11.5 \%$. This wide range could be due to the different populations studied. However, Nilsson and colleagues' experience of a large cohort of adults with epilepsy $(\mathrm{N}=9061)$ is interesting. The authors identified 26 individuals with epilepsy who committed suicide and compared their case histories with those of 171 individuals with epilepsy (controls). They reported a 9-fold increase in the risk of suicide in the context of a mental illness and a 10 -fold increase in relative risk with the use of antipsychotic drugs (Nilsson et al 2002).

Many studies have reported elevated rates of suicide among individuals with epilepsy. In a population-based study, Hauser and colleagues (980) reported only 3 suicides, which was not above the expected number. Cockerell and colleagues (1994), in a population study $(\mathrm{N}=1091)$, reported only 1 suicide during a follow-up of 6.9 (median) years. A group in Iceland (Rafnsson et al 2001) reported 4 suicides among 224 individuals with epilepsy, who were followed up for more than 30 years; this suicide rate was higher than would have been predicted. Recently, two large metaanalyses (Pompili et al 2005, 2006) of 30 studies comprising 51,216 people with epilepsy concluded that suicide in patients with epilepsy is more frequent than in the general population (Pompili et al 2005).

Finally, we should note the methodological issues discussed in a recently published study of mortality among epileptics (Logroscino and Hesdorffer 2005): this study suggests that the gold standard for studies on mortality should be a population-based cohort of incident cases. Only this type of study permits a complete collection of data and the observation of the clinical phase of the disease from the diagnosis of epilepsy to the outcome of the study, death.

\section{Risk factors}

Predicting death caused by suicide is a nearly impossible task. Yet assessing suicide risk requires identification of the factors that make individuals susceptible, and quantifying the individual's level of risk.

\section{Psychiatric comorbidity}

Psychiatric comorbidity is the primary risk factor. Individuals with epilepsy appear to have elevated rates of Axis I disorders according to the DSM, ranging from 19\% to 62\% (Shukla et al 1979; Fiordelli et al 1993; Manchanda et al 1996), with rates of major depressive episodes from $32 \%$ to $48 \%$ (Blumer et al 1997; Robertson 1997; Kanner 1999; Gilliam et al 2002). Nilsson and colleagues (2002) have shown a ninefold increase in related risk of suicide associated with psychiatric illness and a $>10$-fold increase in related risk suicide with the intake of antipsychotic drugs.

Among epileptic patients, the most frequent diagnoses include affective disorders, schizophrenia, and substance abuse. Psychosis in particular may contribute to increased suicide risk: postictal psychotic episodes may increase the risk of suicide in individuals with epilepsy and, more specifically, individuals with temporal lobe epilepsy (TLE) (Mendez et al 1992; Fukuchi et al 2002).

Fukuchi and colleagues (2002) showed that three of six individuals with complex partial seizures who committed suicide did so during postictal psychotic episodes, so that the presence of psychotic symptoms may increase the risk of death by suicide among individuals with epilepsy.

Psychiatric disorders in epilepsy may result from the inhibitory activity that develops as a reaction to the excessive excitatory activity of the chronic seizure disorder (Pompili et al 2005, 2006).

Blumer and colleagues (2002) suggested that successful inhibition of psychiatric disorders could result in persistent suppression of seizures in chronic epilepsy, although some people are at risk of developing many different types of psychiatric complications. The relationship between epilepsy and psychiatric disorders is based on: (1) delay in the development of interictal dysphoric and even more of psychotic disorders after onset of epilepsy, as inhibitory mechanisms become increasingly established; (2) as seizures decrease and are controlled, dysphoric symptoms and psychosis tend to be exacerbated or to emerge de novo (Hill 1953; Blumer 1984; Blumer et al 2000); (3) the same psychiatric changes also appear at times when acute worsening of the seizures causes an enhanced inhibitory response, commonly in the prodromal and postictal phases; (4) a delay of 6-18 months has been noted before psychiatric changes after optimal surgical elimination of the epileptogenetic zone.

The acute engagement of inhibitory mechanisms by the seizure event tends to result in peri-ictal dysphoric symptoms that may include postictal depressive mood with consequent possible suicide.

Reports vary on the prevalence of interictal psychosis, but it is considered higher than the prevalence of psychosis in the general population (Nilsson et al 2002). Kanemoto and colleagues (1999) showed suicide attempts to be more closely associated with postictal psychosis than with interictal psychosis.

Risks for depression in epilepsy include genetic, neurologic, and iatrogenic factors. A family history of depression is quite common among depressed patients with epilepsy. 
Many of these patients report a family history of psychiatric illness, usually mood disorders (Kanner et al 2000).

Epilepsy and depression may share common pathogenic transmitter mechanisms involving decreased serotonergic, noradrenergic, dopaminergic, and GABAergic activity (Kanner et al 2002; Kanner 2005). Seizure type has been shown to correlate neurologically with depression, which is more common in patients with complex partial seizures, particularly those of temporal lobe origin. Most studies have found that depression is more common in those with leftside foci (Mendez et al 1999) suggesting a specific epileptic psycho syndrome (Lambert et al 1986) resulting from limbic dysfunction. A number of antiepileptic drugs have been implicated in the etiology of depression, especially barbiturates (Lambert et al 1986; Plioplys 2003).

A chronic illness is in itself depressing and burdensome (Plioplys 2003). A number of factors contribute: the patient's sense of having no control over seizures, the adverse effects of medication, the illness itself, resentment of a loss of independence seen in the need for life-long medication, fear that the stigma attached to epilepsy will interfere with social relationships, and anxiety about finances and medical insurance. Teenagers and adolescents are especially concerned with issues related to independence (Cramer 1994).

\section{Gender differences}

Although certain gender discrepancies exist in suicidal risk factors, gender seems to be an important factor in predicting suicide. A recent study (Kalinin et al 2005) of male gender showed that the suicidality grade depended on the presence of simple partial seizures (SPS), primary generalized seizures (PGS), secondary generalized seizures (SGS), and complex partial seizure (CPS). A high degree of the first three seizures types determines a high degree of suicidality, while a large value for CPS implies, in contrast, a lower degree of suicidality.

The presence of the above-mentioned seizure types could be a trigger factor in suicide attempt decisions. On the other hand, these seizure types could be connected to mental deterioration, itself a risk factor for suicide (Steinert et al 1994; Kalinin et al 2005).

A large number of generalized tonic-clonic seizures is considered to be a risk factor for the development of cognitive impairment (Dodrill 1986; Aldenkamp 1997).

Certain discrepancies exist between men and women with respect to the basic characteristics of epilepsy and suicide rate. A risk factor for suicide in men is a high frequency of SGS, SPS, and all types of seizures. As most partial seizures have a temporal lobe origin, some authors concluded that temporal lobe epilepsy (TLE) in males represents a risk factor for suicidal behavior. Among female patients, in contrast, the presence of CPS is inversely correlated with death caused by suicide (Kalinin et al 2005).

Early age epilepsy onset means a higher risk of suicide in men but not in women (Kalinin et al 2005). A different pattern of variables was obtained for females, consisting mostly of values of daily doses of certain anti-epileptic drugs (AEDs), that is, phenobarbital PHB, carbamazepine CBZ, valproate VPA, clonazepam CNZ. A high daily dose of PHB and high total number of all seizures would imply a higher degree of suicidality, while the other variables, including daily doses of different AEDs are more ambiguous and less useful for suicidality prediction in women.

The prevalence of AED variables in the female gender can probably be explained by the behavioral toxicity of phenobarbitone (PHB) and lack of toxicity during treatment with carbamazepine (CBZ), valplroic acid (VPA), and clonazepam (CNZ). In addition, PHB is regarded as a depressogenic drug (Reijs et al 2004), and depression, in turn, could trigger suicidal attempts in women.

A further study showed that daily doses of CBZ and VPA were negatively correlated with suicidal behavior especially in females who are more susceptible to depression: perhaps it could depend on their normotimic and antidepressive mode of action (Kalinin et al 2005).

The development of new AEDs has occurred during the past few years, which are now available to treat all forms of epilepsy. Among these is topiramate (Kellett et al 1999), oxcarbazepine (Houtkooper et al 1987), gabapentin (Beydoun et al 1997), lamotrigine (Trenite et al 2001), and levetiracetam (Cramer et al 2000). Unfortunately little information is available on the psychiatric effects of these new AEDs, although probably lamotrigine's antidepressant action might suggest some advantages for its use (Critchley 1994).

The risk of suicide in the presence of depression is fivefold higher in women and only twofold higher in men than in the general population (Kalinin et al 2005a, 2005b).

Concomitant cognitive deterioration and personality traits or so-called "enechetic constitution" and "glishroidia" have also been shown to be significantly related to suicidal risk (Ritaccio et al 2001). Within each gender the presence of cognitive deterioration increases suicide risk approximately fivefold compared with the presence of depression. In other words, cognitive impairment is a much stronger risk factor for suicide than depression in both men and women, whereas 
depression remains a risk factor strictly for women but not for men (Kalinin et al 2005a).

\section{Adolescence}

Adolescence is a period marked by profound biological, social, and psychological developmental changes (Kaplan et al 1998). Depression and other disorders are common problems among adolescents (Baker 2006), but are underdiagnosed. Signs of depression are often dismissed as "normal" reactions in this age group (Lagges et al 2003) and depressed adolescents may appear irritated, bored, and impulsive (Brent et al 2002).

Depression is much more common in adolescents with chronic disease, such as diabetes and epilepsy (Brent et al 2002; Suris et al 1996). A greater proportion of girls with chronic illness report feeling sad, having suicidal thoughts, and other symptoms of depression. In contrast, no significant differences were seen between boys with chronic illness and controls, although there was a trend toward having more emotional problems (Suris et al 1996).

Many studies showed that onset of epilepsy in a period of psychosocial maturation is of greater importance than a long-standing illness, although the precise mechanisms leading to the increased suicide risk are not known and might well be multifactorial (Nilsson et al 2002).

\section{Additional risk factors}

Axis I disorders are a primary risk factor for suicide, although additional risk factors must be considered when conducting a suicide assessment. General risk factors for suicide include amily issues, physical health, personality, life stress, previous suicidal behavior, and access to firearms or other destructive means (Obfaunwa et al 1994).

A family history of suicidal behavior increases the risk of suicide, and the risk increases if coupled with a family history of affective disorder or substance abuse (Moscicki 1997).

Care should be taken to assess an individual's perception of health issues, and how it might influence thoughts about the future. Individuals with epilepsy may be affected by risk factors such as an increase in unemployment rates and financial stress, and possibly increased family stress as a result of complications associated with a chronic medical condition (Robertson 1997; Jones et al 2003; Caplan et al 2005).

Table 1 summarizes the risk factors in epileptic patients.

\section{Assessment}

At the time of the initial diagnostic evaluation, the physician who is managing the epileptic patient should make
Table I Risk factors for death caused by suicide in eplieptic patients

-Psychiatric comorbidity

Axis I

Major depression

Affective disorders

Psychoses

Substance abuse

Axis II

Personality disorders

-Cognitive deterioration

-Antiepileptic drugs

Phenobarbital

-Types of seizures

Temporal lobe epilepsy

Simple partial seizures

Primary generalized seizures

Secondary generalized seizures

-Frequency of seizures

-Early age at epilepsy onset

a psychiatric and psychosocial assessment to identify the patient's risk for suicide. This approach enables the physician to assess the effect of epilepsy on the patient and to detect psychiatric disorder. Effective treatment options include both somatic and non somatic therapies as well as combinations thereof.

Interventions include medications, referral to a psychologist or psychiatrist, or even hospitalization. If appropriate intervention can be introduced, suicide rates may be significantly reduced (Jones et al 2003).

Greater attention is needed to other potentially selfinjurious behaviors such as willful noncompliance with antiepileptic drug (AED) therapy and participation in activities that might be considered dangerous for those with active epilepsy (eg, swimming, climbing) (Jones et al 2003).

A number of methods can be used to screen for suicide risk factors. Jones and colleagues (2003) report a useful instrument developed to assess individuals for psychiatric disorders and suicidality called MINI (Mini-International Neuropsychiatric Interview). The MINI module includes a systematic and useful series of questions that could be included in a clinical interview when screening for depression and psychiatric comorbidity in a busy clinical setting. MINI has a practical scoring system to help quantify current suicide risk and may prove useful in determining the intervention required.

Further self-report measures include the Beck Depression Inventory, which contains suicidal ideation-related items (eg, question 9: "suicide thoughts and wishes") that may prove useful for rapid screening. 
Pharmacotherapy options in epileptic patients with mood disorders include carbamazepine and valproate, considered drugs of first choice for their normothymic mode of action (Kalinin et al 2005). Giving patients AEDs which can cause depression, such as topiramate or levotiracetan must be avoided. Topiramate, in particular, has been associated with suicide among epileptic patients (Rejis et al 2004; Prueter et al 2005; Selai et al 2005).

As well as AED therapy, the antidepressant selective serotonin reuptake inhibitors (SSRIs) can be used to control the affective aspects. However children and adolescents should not be given SSRIs as first-line agents for mild to moderate depression and should be treated with nonsomatic therapies, especially cognitive-behavioral therapy, interpersonal psychotherapy, group therapy, and psychoeducational programs (Baker et al 2006). The Food and Drug Administration (FDA) warns against the use of any SSRIs in children and adolescents, and suggests that only fluoxetine has been shown to be efficacious for these patients (FDA 2005).

\section{Conclusions}

Suicide in epileptic patients is a real problem, frequently underdiagnosed by physicians. Possible risk factors for suicide in all epileptic patients must always be evaluated. When one or more risk factors are discovered, referring physicians and families must try to understand the underlying causes. Few practical approaches are available but a combination of psychiatry, psychoeducational programs, and pharmacotherapy should be tried.

\section{References}

Aldenkamp A. 1997. Effect of seizures and epileptiform discharges on cognitive function. Epilepsia, 38(Suppl 1):S52-5.

Baker GA. 2006. Depression and suicide in adolescents with epilepsy. Neurology, 66(Suppl 3):S5-S12.

Beydoun A, Fischer J, Labar DR, et al. 1997. Gabapentin monotherapy: II. A 26-week, double-blind, dose-controlled, multicenter study of conversion from polytherapy in outpatients with refractory complex partial or secondarily generalized seizures. The US Gabapentin Study Group 82/83. Neurology, 49:746-52.

Blumer D, Montouris G, Davies K, et al. 2002. Suicide in epilepsy: Psychopathology, pathogenesis and prevention. Epilepsy Behav, 3:232-41.

Blumer D, Altshuler L. 1997. Affective disorders. In: Engel J, Pedley TA eds. Epilepsy: a comprehensive textbook. Philadelphia: LippincottRaven, pp. 2083-99.

Blumer D, Wakhlu S, Montouris G, et al. 2000. Treatment of the interictal psychoses. J Clin Psychiatry, 61:110-22.

Blumer D. 1984. Dysphoric disorders and paroxysmal affects: recognition and treatment of epilepsy-related psychiatric disorders. Harv Rev Psychiatry Pr, pp. 271-91.

Brent DA, Birmaher B. 2002. Clinical practice: adolescent depression. NEngl J Med, 347:667-71.

Caplan R, Siddarth P, Suresh G, et al. 2005. Depression and anxiety disorders in pediatric epilepsy. Epilepsia, 46:720-30.

Cockerell OC, Johnson AL, Sander JW, et al. 1994. Mortality from epilepsy: results from a prospective population-based study. Lancet, 344:918-21.
Cramer JA. 1994. Quality of life for people with epilepsy. Neurol Clin, $12: 1-13$.

Cramer JA, Arrigo C, Van Hammee G. 2000. Effect of levetiracetam on epilepsy-related quality of life. N132 Study Group. Epilepsia, 41:868-74.

Critchley MAE. 1994. Effects of lamotigine (Lamictal) in an animal model of anxiety. Br J Pharmacol, 111(Suppl):205P.

Dodrill C. 1986. Correlates of generalized tonic-clonic seizures with intellectual, neuropsychological, emotional, and social function in patients with epilepsy. Epilepsia, 27:399-411.

FDA. 2005. Suicidality in children and adolescents being treated with antidepressant medication. FDA Public Health Advisory, October 15, 2004 [online]. Accessed November 10, 2005. URL: http://www.fda. gov/cder/drug/antidepressants/SSRIPHA200410.htm.

Fiordelli E, Beghi E, Bogliun G, et al. 1993. Epilepsy and psychiatric disturbance: a cross-sectional study. Br J Psychiatry, 163:446-50.

Fukuchi T, Kanemoto K, Masaaki K, et al. 2002. Death in epilepsy with special attention to suicide cases. Epilepsy Res, 51:233-6.

Gilliam F, Kanner AM. 2002. Treatment of depressive disorders in epilepsy patients. Epilepsy Behav, 3:2-9.

Goodwin FK, Jamison KR. 1990. Manic-depressive illness. Oxford: Oxford University Press.

Hauser WA, Annegers JF, Elveback LR. 1980. Mortality in patients with epilepsy. Epilepsia, 21:399-412.

Henriksen B, Juul-Jensen PP, Lund M. 1970. The mortality of epileptics. In: Brackenridge RDC, editor. Life assurance medicine. London: Pitman, pp. 139-48.

Hill D. 1953. Psychiatric disorders of epilepsy. Med Press, 113:522-8.

Houtkooper MA, Lammertsma A, Meyer JW. 1987. Oxcarbazepine (GP 47.680): a possible alternative to carbamazepine? Epilepsy, 28:693-8.

Jones JE, Hermann BP, Barry JJ, et al. 2003. Rates and risk factors for suicide, suicidal ideation, and suicide attempts in chronic epilepsy. Epilepsy Behav, 4:S31-S8.

Kalinin VV, Polyanskiy DA. 2005a. Gender differences in risk factors of suicidal behaviour in epilepsy. Epilepsy Behav, 6:424-9.

Kalinin VV, Polyanskiy DA. 2005b. Gender and suicidality prediction in epilepsy. Epilepsy Behav, 7:657-63.

Kanemoto K, Kawasaki J, Mori E. 1999. Violence and epilepsy: a close relation between violence and postictal psychosis. Epilepsia, 40:107-9.

Kanner AM. 2005. Depression in epilepsy: a neurobiologic perspective. Epilepsy Cur, 5:21-7.

Kanner AM, Balabanov A. 2002. Depression and epilepsy: how closely related are they? Neurology, 58:s27-39.

Kanner A, Nieto J. 1999. Depressive disorders in epilepsy. Neurology, 53:26-32.

Kanner AM, Palac S. 2000. Depression in epilepsy: a common but often unrecognized comorbid malady. Epilepsy Behav, 1:37-51.

Kaplan HI, Sadock BJ. 1998. Kaplan and Sadock's synopsis of psychiatry. 8th ed. Philadelphia: Lippincott Williams and Wilkins.

Kellett MW, Smith DF, Stockton PA. 1999. Topiramate in clinical practice: first year's postlicensing experience in a specialist epilepsy clinic. J Neurol Neurosur Psychiatry, 66:759-63.

Lagges AM, Dunn DW. 2003. Depression in children and adolescents. Neurol Clin, 21:953-60.

Lambert MV, Robertson MM. 1999. Depression in epilepsy: etiology, phenomenology and treatment. Epilepsia, 40(Suppl 10):S21-47.

Logroscino G, Hesdorffer DC. 2005. Methodologic issues in studies of mortality following epilepsy: measures, types of studies, sources of cases, cohort effects, and competing risks. Epilepsia, 46:3-7.

Manchanda R, Schaefer B, McLachlan R. 1996. Psychiatric disorders in candidates for epilepsy surgery. J Neurol Neurosurg Psychiatry, 61:82-9.

Mendez DF, Cumming JL, Benson DF. 1986. Depression in epilepsy: significance and phenomenology. Arch Neurol, 43:766-70.

Mendez MF, Doss RC. 1992. Ictal and psychiatric aspects of suicide in epileptic patients. Int J Psychiatry Med, 22:231-7. 
Mos'cicki EK. 1997. Identification of suicide risk factors using epidemiologic studies. Psychiat Clin North Am, 20:499-517.

Murphy GE. 1994. Suicide and attempted suicide. In: Winokur G, Clayton PJ eds. The medical basis of psychiatry. Philadelphia: Saunders, pp. 529-44.

Nilsson L, Ahlbom A, Farahmand BY, et al. 2002. Risk factors for suicide in epilepsy: a case control study. Epilepsia, 43:644-51.

Obafunwa JO, Busuttil A. 1994. Clinical contact preceding suicide. Postgrad Med J, 70:428-32.

Ott D, Siddart P, Gurbani S, et al. 2003. Behavioral disorders in pediatric epielpsy: unmet psychiatric need. Epilepsia, 44:591-7.

Plioplys S. 2003. Depression in children and adolescents with epilepsy. Epilepsy Behav, 4:S39-S45.

Pompili M, Girardi P, Ruberto A, et al. 2005. Suicide in the epilepsies: a meta-analytic investigation of 29 cohorts. Epilepsy Behav, 7:305-10.

Pompili M, Girardi P, Tatarelli R. 2006. Death from suicide versus mortalità from epilepsy in the epilepsies: A meta-analysis. Epilepsy Behav, 9:641-8.

Prueter C, Norra C. 2005. Mood disorders and their treatment in patients with epilepsy. J Neuropsychiatry Clin Neurosci, 17:20-8.

Rafnsson V, Olafsson E, Hauser WA, et al. 2001. Cause-specific mortality in adults with unprovoked seizures: a population-based incidence cohort study. Neuroepidemiology, 20:232-6.
Rejis R, Aldenkamp AP, De Krom M. 2004. Mood effect of antiepileptic drugs. Epilepsy Behav, 5(Suppl 1):S66-76.

Ritaccio A, Devinsky O. 2001. Personality disorders in epilepsy. In: Ettinger AB, Kanner AM, eds. Psychiatric issues in epilepsy: a practical guide to diagnose and treatment. Philadelphia: Lippincott Williams and Wilkins, pp. 147-61.

Robertson M. 1997. Depression in neurological disorders. In: Robertson MM, Katona CLE eds. Depression and physical illness. Chichester: Wiley, pp. 305-40.

Robertson MM, Trimble MR, Townsend HRA. 1999. Phenomenology of depression in epilepsy. Epilepsia, 28:364-72.

Selai C, Bannister D, Trimble M. 2005. Antiepileptic drugs and the regulation of mood and quality of life (QOL): the evidence from epilepsy. Epilepsia, 46(Suppl 4):50-7.

Shukla G, Srivastava O, Katiyar B, et al. 1979. Psychiatric manifestation in temporal lobe epilepsy: a controlled study. $\mathrm{Br} J$ Psychiatry, 135:411-17.

Steinert T, Froscher W. 1994. Aggression bei Epilepsie. Nervenheikd, 13: s199-205.

Suris JC, Parera N, Puig C. 1996. Chronic illness and emotional distress in adolescence. $J$ Adolesc Health, 19:153-6.

Trenite DG, Rentmeester TW, Scholtes FB. 2001. Perimarketing surveillance of lamotrigine in The Netherlands: doctor's and patients' viewpoints. Pharm World Sci, 23:1-5. 